\title{
PARES REVISORES
}

Hamut'ay 3(2). Julio-diciembre 2016

Dra. Teresa Piñeiro Otero

Facultad de Ciencias de la Comunicación,

Universidade da Coruña, España

Dra. Esperanza Valero Rueda

Docente - Investigadora Educativa en el área de Pedagogía, currículo y TIC, Gestión de la tecnología en la Educación Superior, Instituto Superior de Ciencias Educativas. Porto-Portugal

\section{Ing. Amaury Cabarcas Álvarez}

Coordinador Programa Ingeniería de Sistemas a Distancia, Docente investigador, Facultad de Ingeniería, Universidad de Cartagena, Colombia

\section{Ing. Norma León Lescano}

Coordinadora de la Unidad de Proyectos de Investigación de la Escuela Profesional de Ingeniería de Computación

y Sistemas, Facultad de Ingeniería y Arquitectura,

Universidad de San Martín de Porres, Perú

\section{Ing. Nancy Esperanza Olarte López}

M.Sc. Tecnologías de la Información Aplicadas a la Educación, Esp. Tecnologías de la Información Aplicadas a la Educación, Docente Planta Programa Tecnología en

Electrónica y Comunicaciones,

Universidad Militar Nueva Granada, Colombia

\section{Dra. Sandra Muñoz Maldonado}

Docente investigadora en Psicología y tecnologías, Sistema de Universidad Abierta y a Distancia, FES Iztacala,

Universidad Nacional Autónoma de México

\section{Dra. Melba Stanziola}

Docente investigadora, Facultad de Psicología, Psicóloga Clínica, Universidad de Panamá

\section{Ing. Carlos Antonio Orrego Muñoz}

M.Sc. Ingeniería de Sistemas Electrónicos. M.Sc. Ingeniería Biomédica. Esp. Gerencia Integral de las Telecomunicaciones. Esp. Interventoría de Proyectos de Telecomunicaciones. Docente de Tiempo Completo,

Universidad Militar Nueva Granada, Colombia

Dra. Belinda Izquierdo García

Investigadora de la Universidad Veracruzana, México

\section{Mag. Violeta Cuenca Cartagena}

Docente Investigador Universidad Cesar Vallejo, Perú
Dr. Víctor Hernández Rivero

Dpto. Didáctica e Investigación Educativa,

Universidad de La Laguna, Espańa

\section{DSC. PhD. Rubén Dario Cárdenas Espinosa}

Ingeniería Electrónica, Tecnología de Información, Líder SENNOVA, Investigador. Director Grupo de Investigación Electrónica, Automatización y Energías Renovables. Instructor SENA Regional Caldas Centro de Automatización Industrial. Catedrático en los CREAD

Dorada, Anserma, Pereira y Manizales,

Universidad de Caldas, Colombia

Dr. Javier Fombona Cadavieco

Docente investigador, Facultad de Formación del Profesorado y Educación, Universidad de Oviedo, España

\section{Mg. John Alexander Rojas Montero}

Departamento de Tecnología - Universidad Pedagógica

Nacional, Docente investigador en el área TIC y educación, Director Grupo de Investigación KENTA. Bogotá, Colombia. 